Protein dynamics

\section{Hard facts on soft centres}

\section{Peter Artymiuk}

LAST YEAR, Doucet and Benoit published in Nature a pioneering paper ${ }^{1}$ on the interpretation of the diffuse scattering maxima in the background of X-ray diffraction patterns from protein crystals. They showed that, as in crystals of small molecules, it is possible to analyse some aspects of this diffuse scattering in terms of correlated molecular displacements. Their work, and that of Phillips et al. ${ }^{2}$, provides a tantalizing glimpse of the wealth of information concerning the dynamical behaviour of biological macromolecules that could, in principle, be extracted from this seemingly intractable source of data (see my recent News and Views article ${ }^{3}$ ). But it seemed that it might be some time before generally applicable methods could be derived which could be used to obtain information of direct biochemical interest from these data. On page 659 of this issue 4 , however, Caspar, Clarage, Salunke and Clarage present a fascinating study of diffuse X-ray scattering, in which they derive evidence concerning both inter- and intra-molecular correlated displacements in crystals of insulin.

It is now generally accepted that coordinated molecular motions and molecular flexibility are crucial to the function of biological macromolecules, and that a full appreciation of the dynamics of proteins and nucleic acids is essential to elucidate their properties. The results obtained by Caspar et al. can be compared directly with, and indeed complement, the information that is available from spectroscopic methods, from moleculardynamics simulations ${ }^{5}$, and from crystallographic temperature factors ${ }^{6}$.

A key step in their interpretation is the identification of the underlying distribution of the scattering with a commonly used crystallographic self-correlation function called the Patterson function. Information about the range and amplitude of coupled displacements within the structure is obtained by theoretical calculations of the diffuse scattering, modified by different range and amplitude parameters. These results are then compared with the experimentally observed patterns.

Caspar et al. identify two distinct types of coupled motion in insulin, each giving rise to a characteristic type of diffuse (or, as they call it, variational) scattering. First, after removing the effects of incoherent (Compton) scattering and the scattering resulting from disordered water, they show that the remaining general diffuse background arises from short-range correlated displacements (with r.m.s. amplitude $0.4 \AA$ ) of sidechains or parts of the protein background over a range of $4-8 \AA . \AA$. These are the kinds of motions that have had to be postulated to explain various processes in biological macromolecules, for example, to explain how oxygen can reach the haem group in myoglobin through a seemingly impenetrable mass of closely packed side-chain atoms $s^{5}$. Caspar et al. show that the second aspect of the diffuse scattering analysed,

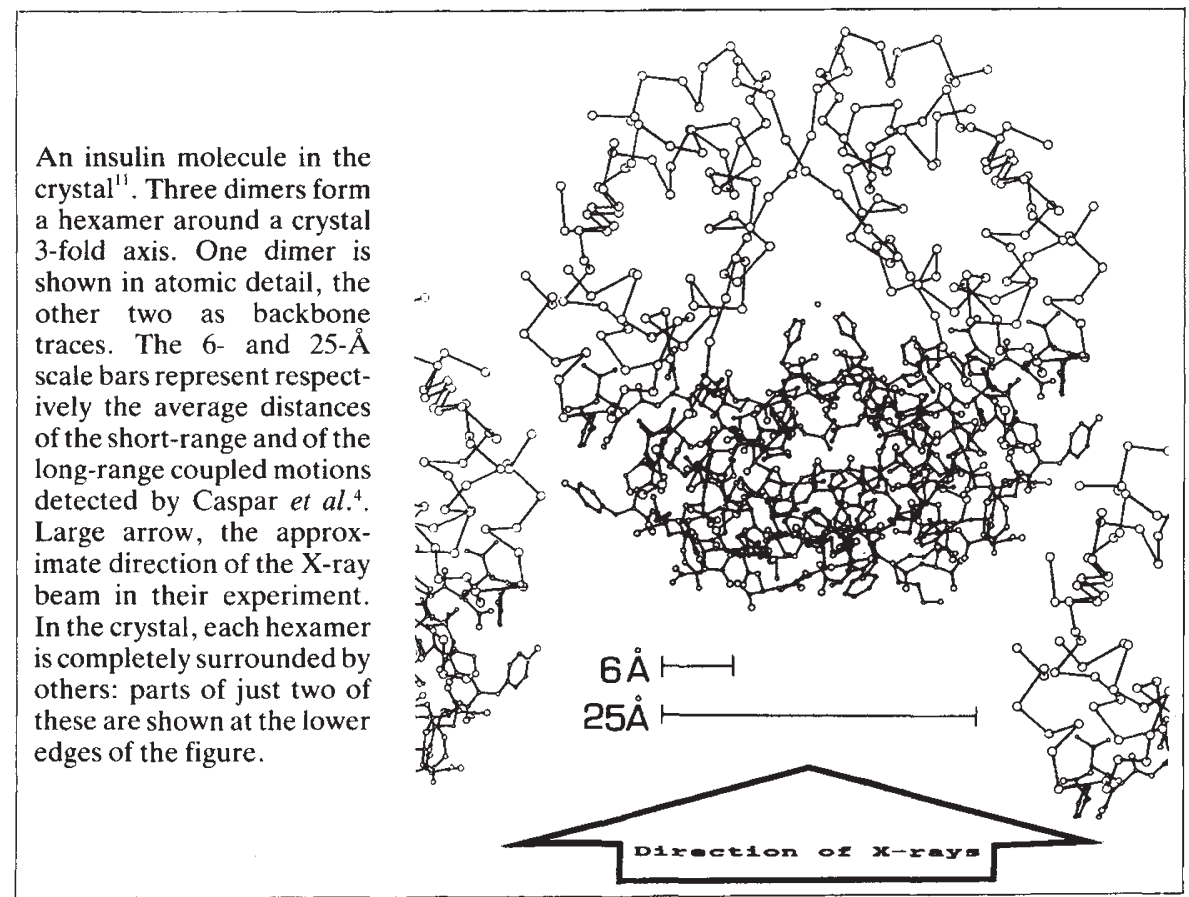

which consists of sharper haloes around the ordinary Bragg diffraction peaks, arises from displacements (coupled over a range of $20-30 \AA$ ) of insulin molecules relative to one another within the crystal. This result is very exciting, as in favourable cases such studies could be extended to correlated motion of subunits in allosteric proteins such as haemoglobin or aspartate transcarbamoylase ${ }^{8}$, where large-scale movements are known to occur between $R$ and $T$ states. In their pioneering study ${ }^{9}$, Boylan and Phillips analysed diffuse scattering from tropomyosin crystals in terms of large-scale anisotropic motions and attempted to relate their results to the function of the protein in muscle.

There are several ambiguities inherent in the interpretation of the diffuse scattering data. The most important of these is the complete absence of information concerning timescales of the 'motions' detected by crystallographic methods in general $^{3}$ : this is why the neutral word 'displacements' is usually used, so that the possibility of static disorder can be included as well. However, much of the information obtained from crystallographic temperature factors is consistent with interpretation in terms of atomic motions $^{10}$, and this could well also be true of much diffuse scattering data'. Another ambiguity in the diffuse-scattering study ${ }^{4}$ of Caspar et al. is that, whereas it is possible to say that there are coupled motions of atoms separated by $4-6 \AA$, and of insulin molecules $20-30 \AA$ apart, it is not possible to say precisely which parts of the structure are involved. At present Caspar et al. base their analyses on one still photograph, but they have already begun to extend their studies into three dimensions by the use of area-detector data ${ }^{4}$. This, together with the evidence from the crystallographic temperature factors, should help resolve these questions.

It is clear that the elegant methods derived by Caspar et al. should lead to much more hard information becoming available about concerted displacements of protein subunits - and of liquid-like movements of side-chains and main-chain segments - both on the surface and in the centre of the protein.

Doucet, J. \& Benoit, J.P. Nature 325, 643-646(1987)

Phillips, G.N. et al. Biophys. J. 32, 485-502 (1980)

Artymiuk, P. Nature 325, 575-576 (1987).

4. Caspar, D.L.D., Clarage, J., Salunke, D.M. \& Clarage, M. Nature 332, 659-662 (1988).

. Karplus, M. \& McCammon, J.A. A. Rev. Biochem. 53 $263-300(1983)$.

6. Artymiuk, P. et al. Nature 280, 563-568 (1979)

7. Perutz M.F Scient. Am 239(6) 68-86 (1978)

8. Krause, K. L. et al. Proc. natn. Acad. Sci. U.S.A. 82, $726-$ $739(1970)$.

9. Boylan, D. \& Phillips, G.N. Biophys. J. 49, 76-78 (1986)

10. Petsko, G.N. \& Ringe, D.A. A. Rev. Biophys. 13, 331 $371(1984)$

11. Dodson, E.J. et al. Can. J. Biochem. 57, 1200-1203 (1987).

Peter Artymiuk is in the Department of Biochemistry, University of Sheffield, Sheffield S10 $2 T N, U K$. 